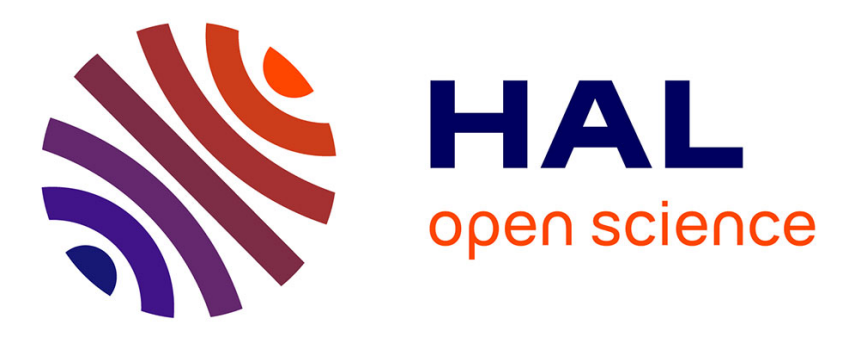

\title{
Broadband tunable lossy metasurface with independent amplitude and phase modulations for acoustic holography
}

Shi-Wang Fan, Yifan Zhu, Liyun Cao, Yan-Feng Wang, A- Li Chen, Aurélien Merkel, Yue-Sheng Wang, B. Assouar

\section{To cite this version:}

Shi-Wang Fan, Yifan Zhu, Liyun Cao, Yan-Feng Wang, A- Li Chen, et al.. Broadband tunable lossy metasurface with independent amplitude and phase modulations for acoustic holography. Smart Materials and Structures, 2020, 29 (10), pp.105038. 10.1088/1361-665X/abaa98 . hal-03043193

\section{HAL Id: hal-03043193 https://hal.science/hal-03043193}

Submitted on 7 Dec 2020

HAL is a multi-disciplinary open access archive for the deposit and dissemination of scientific research documents, whether they are published or not. The documents may come from teaching and research institutions in France or abroad, or from public or private research centers.
L'archive ouverte pluridisciplinaire HAL, est destinée au dépôt et à la diffusion de documents scientifiques de niveau recherche, publiés ou non, émanant des établissements d'enseignement et de recherche français ou étrangers, des laboratoires publics ou privés. 


\title{
Broadband tunable lossy metasurface with independent amplitude and phase modulations for acoustic holography
}

\author{
Shi-Wang Fan ${ }^{1,2}$, Yifan Zhu ${ }^{2}$, Liyun Cao $^{2}$, Yan-Feng Wang ${ }^{3}$, A-Li Chen ${ }^{1}$, Aurélien Merkel ${ }^{2}$, Yue-Sheng \\ Wang $^{1,3, *}$, Badreddine Assouar ${ }^{2, *}$ \\ ${ }^{1}$ Institute of Engineering Mechanics, Beijing Jiaotong University, Beijing, 100044, China \\ ${ }^{2}$ Institut Jean Lamour, CNRS, Universite de Lorraine, Nancy, 54000, France \\ ${ }^{3}$ Department of Mechanics, School of Mechanical Engineering, Tianjin University, Tianjin, 300350, China \\ *Corresponding authors.E-mail addresses:yswang@tju.edu.cn and badreddine.assouar@univ-lorraine.fr
}

\begin{abstract}
Metasurface-based acoustic hologram projectors fabricated with fixed microstructures can only generate the predesigned images at a single or few discrete frequencies. Here, a variety of acoustic holographic applications can be realized in broadband by a matched helical design of the tunable lossy acoustic metasurface (TLAM). The proposed TLAM unit is composed of a grating channel and an adjustable internal absorber to achieve the independent amplitude and phase modulations (APM) in a continuous frequency range. We demonstrate the excellent performance of the scattering-free anomalous refection by the APM method for tuning loss without foam materials. Then, the multi-plane acoustic holograms and the broadband holographic images are demonstrated by the flexible reconfigurations of one designed TLAM. Due to the compact design and the great flexibility, this proposal may be more practical to achieve the high-quality holograms with multi-scale fine manipulation and multiplexed acoustic communication with high information content.
\end{abstract}

Keywords acoustic metamaterials, metasurfaces, holography, independent modulation, continuous tunability 


\section{Introduction}

Acoustic holography (AH) has emerged to reconstruct and visualize the desired complex sound fields [1], which offers excellent capabilities for ultrasonic treatment [2] and particle manipulation [3, 4]. The direct approach to realize $\mathrm{AH}$ normally relies on the phased source arrays with many individually controlled transducer [2-4]. These sources can actively modulate the imaging fields, but is also usually bulky and costly, and needs careful calibration circuit systems. Subsequently, as a class of artificial metamaterials [5], acoustic metasurfaces (AMs) [6-8] have been proposed to manipulate the uniform signal from a single source, and passively implement the low-cost acoustic holograms with ultrathin structure thickness [9-15]. The conventional AM approaches for $\mathrm{AH}$ images based on the single phase modulation (SPM) $[9,10]$ are very complicated for optimization, and result in significant clutter outside the controlled region for many target fields. Recently, AMs with independent amplitude and phase modulations (APM) [11-15] have been used to generate high-fidelity acoustic holograms. In the context of complete acoustic modulation, both amplitude and phase are simultaneously manipulated [16-19]. The additional degree of freedom leads to a marked simplification of the $\mathrm{AH}$ design process. The $\mathrm{AH}$ may be realized by back-propagating the target field to the structure plane and then creating the conjugate field over this surface (i.e., time-reversal technique) [20], instead of using the complex optimization algorithms.

However, the above AMs with APM method can only operate at one [11-13] or four [14] designed 
frequencies, because they are typically predefined by their geometries and cannot be changed once the devices were manufactured. Recently, an asymmetric coding metasurface for the controllable projection of acoustic images was designed and realized experimentally [15]. The switching mode of the coding elements can be achieved by using the asymmetric incident waves. No investigation on tunable [21-25], reconfigurable [26-30], or programmable [31-33] AMs to realize broadband AH has been reported up to now. In addition, the porous foams are used at the end of the fixed AM structures to absorb the leaky sound waves $[12,14]$, which may be an uncertainty in terms of sound losses.

In this work, we propose the design of a tunable lossy acoustic metasurface (TLAM) that simultaneously realizes APM and broadband without foams. Thus, the AH process can generally project arbitrarily predesigned images operating within a continuous frequency range, rather than at an isolated or discrete frequencies. The proposed TLAM unit is composed of a grating channel and an adjustable internal absorber with the quarter-wavelength resonance. One of the tunable parameters is separated to modulate the amplitude loss independently to achieve the APM. The theoretical analysis and the numerical simulation are performed to obtain the arbitrary combinations of phases and amplitudes. Then, they are applied to design a flat TLAM for scattering-free anomalous reflection, which can reflect an incident plane wave along the desired direction without parasitic scattering in any unwanted direction. We furthermore extend the tunability for three-dimensional (3D) TLAM to generate the high-fidelity AH images in multiple planes or at different frequencies. 
This can be more practical by using the same TLAM to continuously engineer the sound fields.

\section{Analytical mechanism for APM design}

Although the purpose of this work is to design a 3D tunable unit and array it into two-dimensional (2D) metasurface, for simplicity while without losing generality to illustrate the principle, we first focus on a very simple 2D lossy unit of which the geometric schematic is inserted in the middle of figure 1(c). The unit consists of four uniformly distributed gaps with width $w$ and length $l$.
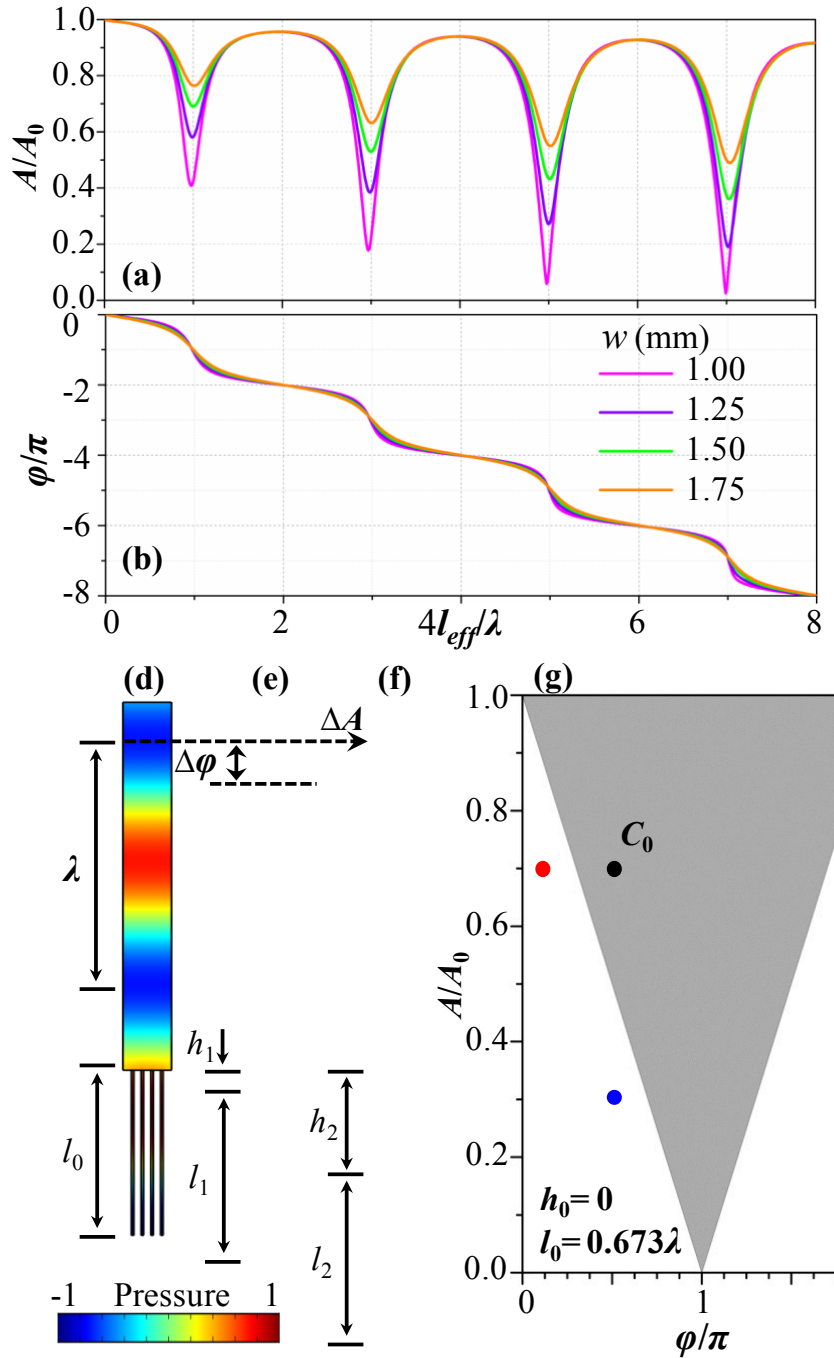

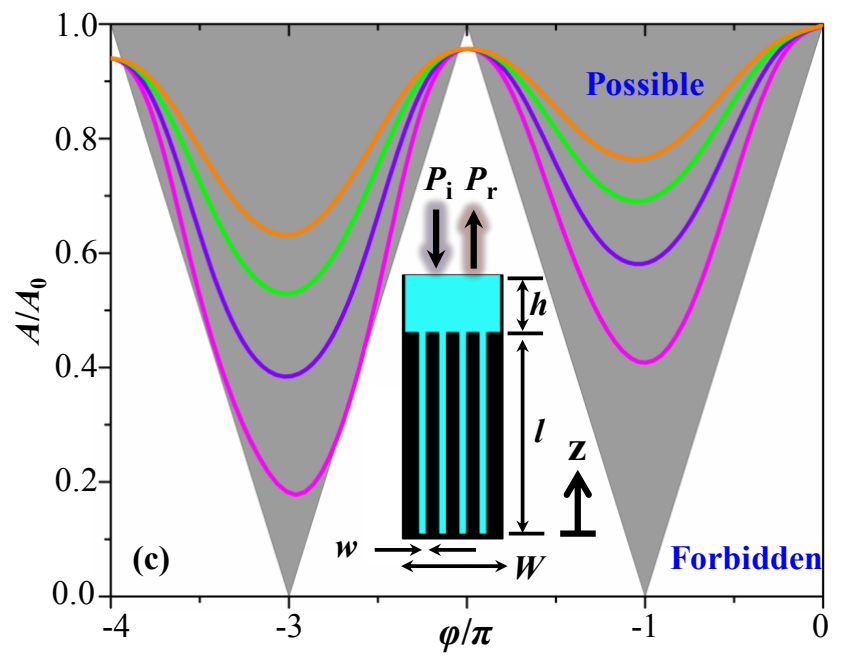

(h)
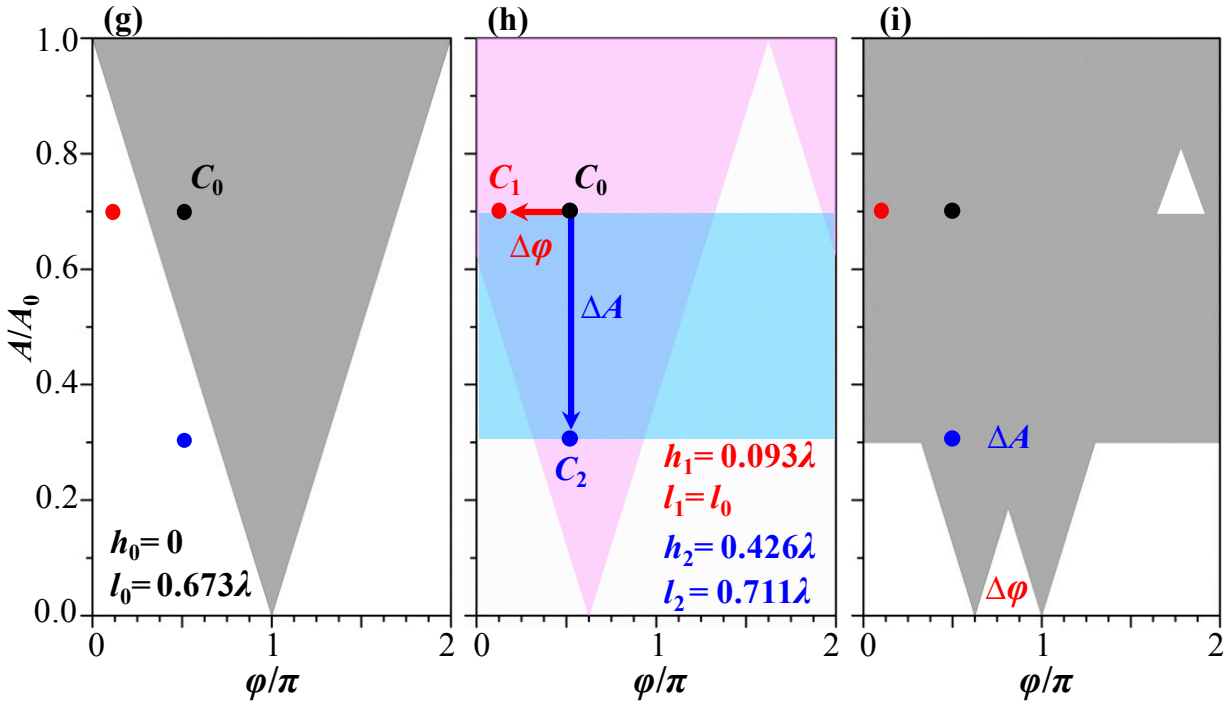

Figure 1. The pressure fields including the reflected amplitude and the phase shift for the analytical 
interpretations of a lossy unit with a quarter-wavelength resonator. (a) The amplitude spectrum of the unit with four different gap widths. (b) The corresponding phase spectrum. (c) The amplitude and phase ranges. The gray regions are the analytically possible range for combinations of phases and amplitudes, whereas the rest (white) regions denote the forbidden range. The illustration in (c) presents the topological schematic geometry of the unit. (d), (e) and (f) are the pressure fields for three different units. (g) The phase and amplitude ranges corresponding to the initial structure in (d). (h) The possible range of values corresponding to the structures in (e) and (f). (i) The merged region of (g) and (h). Black, red and blue dots mark the positions for the three cases.

A plane acoustic wave propagating into the gaps along the $z$ axis can be written as $P_{\mathrm{i}}=p_{0} \exp (i k z)$ with $k=2 \pi / \lambda$ being the wave number in which $\lambda=c_{0} / f$ is the wavelength with $f$ being the incident frequency and $c_{0}$ the sound velocity. At the closed end of the gaps, total reflection occurs and the reflected wave is denoted as $P_{\mathrm{r}}=p_{0} \exp (-i k z)$. Therefore, the total pressure in the gaps is the superposition of the incident waves and their reflections, which is expressed as $P_{\mathrm{t}}=P_{\mathrm{i}}+P_{\mathrm{r}}=2 p_{0} \cos (k z)$. It should be noticed that the total pressure field in the air gaps is a standing wave field. If the wave node of the standing wave is located at the open end of the gaps, i.e., $P_{\mathrm{t}}=0$ at $z=l$, then $k l=(2 n-1) \pi / 2$ and the sound will not leak out by re-radiation. As a result, the efficient gap length must satisfy the following requirement as:

$$
l_{\text {eff }}=(2 n-1) \lambda / 4, \quad n=1,2,3 \ldots
$$

where $n$ is a positive integer and $l_{\text {eff }}=l+\Delta l$ in which $\Delta l=8 w / 3 \pi$ represents the end correction caused by the radiation mass at the open end of the gaps [34]. This means that the efficient gap length with 
an odd number integer multiple of quarter-wavelength can guarantee the perfect trapping the sound in the gap, which is also known as the quarter-wavelength resonance [35-39].

Alternatively, we can also interpret this effect by the impedance transfer equation. The definitions of the acoustic impedances $Z_{\mathrm{cl}}$ at $z=0$ (closed end) and $Z_{\mathrm{op}}$ at $z=l$ (open end) can be made in the background medium of air with the mass density $\rho_{0}$. Hence the input impedance of the resonator can be expressed as:

$$
Z_{\text {op }}=Z_{\text {a }} \frac{Z_{\mathrm{cl}}+i Z_{\mathrm{a}} \tan (k l)}{Z_{\mathrm{a}}+i Z_{\mathrm{cl}} \tan (k l)},
$$

where $Z_{\mathrm{a}}=\rho_{0} c_{0} / 4 w$ is the characteristic impedance of the air gaps. Because the impedance of the rigid closed end is considered infinitely large, the input impedance reduces to $Z_{\mathrm{op}}=-i Z_{a} \cot (k l)$. As long as $k l=(2 n-1) \pi / 2$, we have $Z_{\mathrm{op}}=0$. Acoustically, a zero impedance corresponds to an acoustic short circuit phenomenon. In other words, the sound wave can radiate into the gaps without any restriction.

In this model, the width $w$ of the gaps is set in the millimeter order, which is very small in comparison to $\lambda$ in kilohertz range. The viscous loss is significant, which results in a high absorption. Based on the above resonant trapping effect, the quasi-perfect absorption can be realized by exploiting the inherent viscosity effect in the background medium of air, without involving any extra sound-absorption foam materials.

Considering the viscosity of the medium in narrow gaps, the attenuation coefficient of sound is approximately [34-36] 


$$
\alpha \approx \frac{2}{w c_{0}} \sqrt{\frac{\eta \omega}{2 \rho_{0}}},
$$

where $\eta$ is the dynamic viscosity coefficient. For the purpose of acquiring a clearer physical mechanism of the underlying design principles, we further analyze the relationship between the reflected fields (including amplitude and phase) and the geometric parameters by combining the finite element method based on COMSOL Multiphysics 5.5 software. The unit width and the gap length are $W=20 \mathrm{~mm}$ and $l=80 \mathrm{~mm}$, respectively. The parameter $h=0$ is excluded in the initial state. We calculate the amplitudes $A$ (normalized by the incident amplitude $A_{0}$ ) and the phases (normalized by $\pi$ ) for the reflected waves as functions of wavelength $\lambda$ (normalized by $l_{\text {eff }}$ ) at four different gap widths $(w)$ and plot the results with colored solid lines in figures 1(a) and (b), respectively. The different gap widths are equivalent to changing the acoustic impedance. One can clearly observe that the strong absorption (i.e., low reflected amplitude) occurs at the quarter-wavelength resonance point where the phase equals to $(1-2 n) \pi$. In physics for transmission, this phenomenon is known as the Fabry-Pérot (FP) resonances which always lead to a full amplitude regardless of the impedance, bringing a constraint of amplitudes and phases as the so-called "FP binding" [16, 40]. We further give the possible range of values for combinations of phases and amplitudes by reducing the impedance as shown by the gray region in figure 1(c), in which the colored solid lines correspond to the results in figures 1(a) and (b), and the rest white regions denote the forbidden amplitudes. Then, we expect to find a way to fill up the forbidden ranges in the combinations and achieve the APM in a 
broadband range.

To clearly show the expanding coverage of the phase-amplitude diagram, we choose three typical units and plot the reflected pressure fields, as shown in figures 1(d), (e) and (f), respectively. Their combinations of the amplitudes and phases (reduced in $2 \pi$ range) are $A=0.7$ and $\varphi=\pi / 2$ for the initial case of $C_{0}, A=0.7$ and $\varphi=\pi / 8$ for $C_{1}$, and $A=0.3$ and $\varphi=\pi / 2$ for $C_{2}$.

For the initial case, the structure can be an arbitrary one of the units with $h_{0}=0$ as we discussed above. When we choose $l_{0}=0.673 \lambda$, the pressure field shows a reflected amplitude 0.7 with phase $\pi / 2$. The gray region in figure $1(\mathrm{~g})$ is the same as a half in figure $1(\mathrm{c})$. The black dot marks the combination for $C_{0}$ as a reference. At point $C_{1}$, other geometric parameters are the same to those in $C_{0}$, but an extra space of the air layer is added with the depth $h_{1}=0.093 \lambda$. Then, the amplitude 0.7 is also observed because the absorption gaps has not been changed and the specific amount of the sound attenuation is remained. However, the reflected phase has a shift to $\pi / 8$ because the phase at the output surface of the unit has decreased due to the extra air layer, as shown by the red arrow in figure 1(h). As a result, the corresponding possible range for $C_{1}$ is shown by the magenta region in figure 1(h), which has the same geometry as that of $C_{0}$, but with the region shifting $2 k h_{1}$ to the left side. For modulating the amplitude from $C_{0}$ to $C_{2}$, the length of gaps are elongated up to $l_{2}=0.711 \lambda$ to move further away from the FP resonance, meanwhile the phase also will be changed, as shown by the cyan region in figure $1(\mathrm{~h})$. Thus, we also adjust the depth of the air layer into $h_{2}=0.426 \lambda$ to match 
the required phase value, because the amplitude is not affected by the depth. The blue dots mark the combination for $C_{2}$, whose amplitude decreases to 0.3 with the same phase $\pi / 2$ as $C_{0}$, as shown by the blue arrow in figure 1(h). Therefore, a particular amplitude can be independently combined with other phases by changing the length of the gaps and the air layer depth.

By extension, the possible range for combination of phases and amplitudes will expand due to the additional choices from $C_{0}$ to $C_{1}$ and $C_{2}$, as shown by the gray region in figure 1(i), which is merged from the regions in figures $1(\mathrm{~g})$ and $(\mathrm{h})$. Similarly, if the depth $h$ can be any value in the range of $\lambda / 2$ and $l$ in the range of $\lambda / 4$, the merged range will cover the entire range of values including the phase change of $2 \pi$ and amplitude change of 1 at any frequency in principle. That means that the arbitrary combination of phases and amplitudes is available to achieve the APM as long as the design of the double-parameter tunable AM structure within a certain frequency range.

\section{The tunability design}

The proposed concept of the broadband TLAM for the AH is presented in figure 2(a). As illustrated, the incident waves in a frequency range can be reflected by the TLAM from the structure plane and projected onto the image plane at a given distance. Figure 2(b) shows that the TLAM unit consists of a grating channel (blue) and an adjustable internal absorber. For the absorber design, the matched screw-and-nut mechanism $[22,27,41]$ is used to significantly compress the thickness of the helical gap (brown). The absorber is constructed by a matched helix (green) rotated into the helical 
gap from bottom to top, and then it can move up and down as a whole along the grating channel.

Thus, there are two tunable parameters (i.e., the rotational angle $r$ of the matched helix and the varying height $h$ of the whole absorber) in the individual unit. Note that the relatively obvious rotational operation of the matched helix can characterize the small variation of the gap length, so the matched helical design can decrease the adjustable system sensitivity to reduce the tuning error [22, 23].

(a)

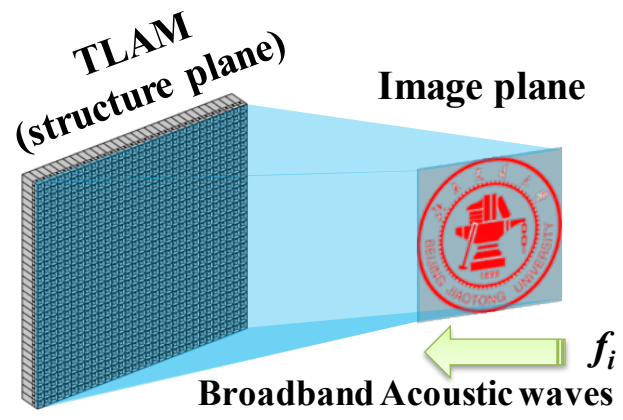

(b)
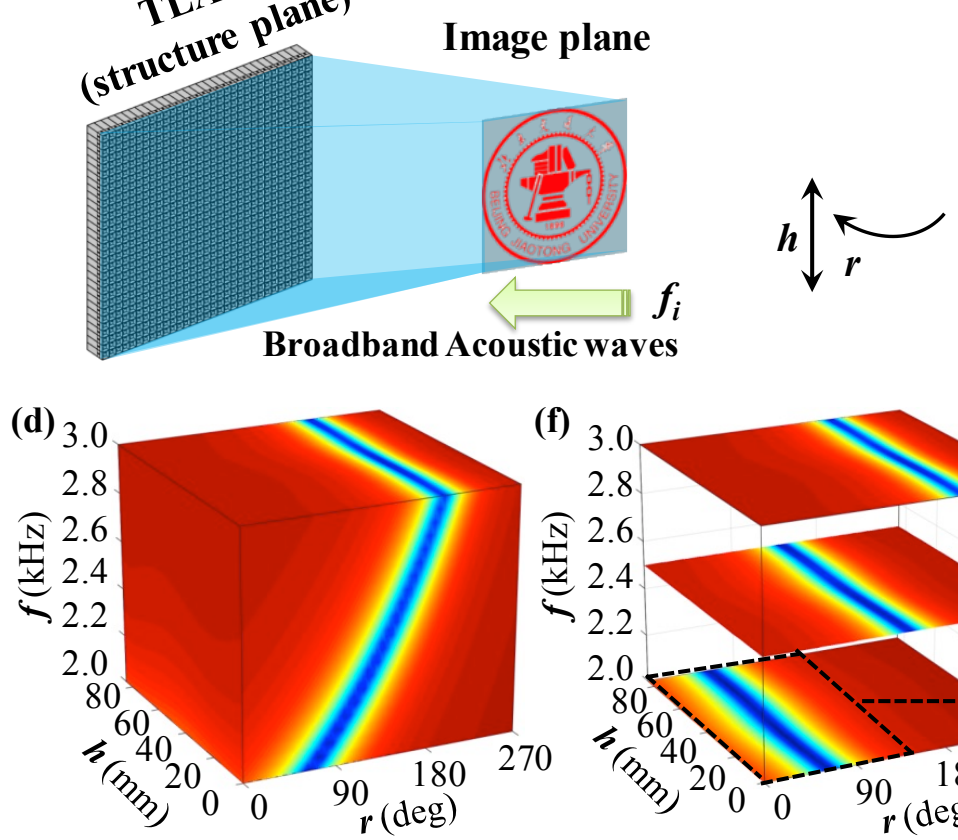

(e)

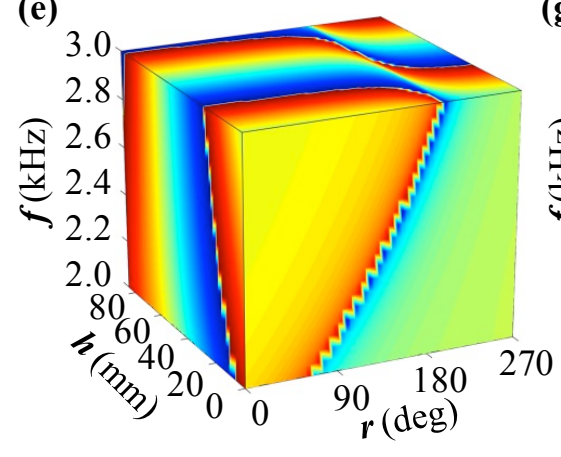

(f) 3.0

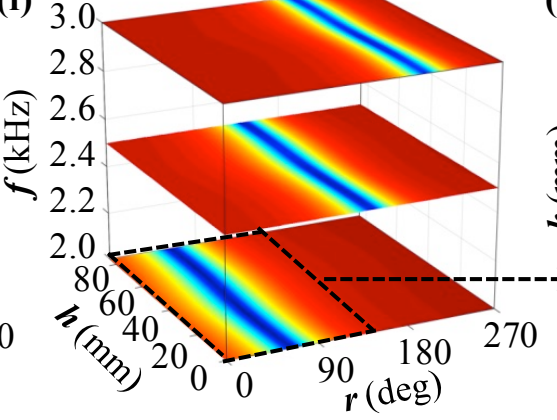

(g)

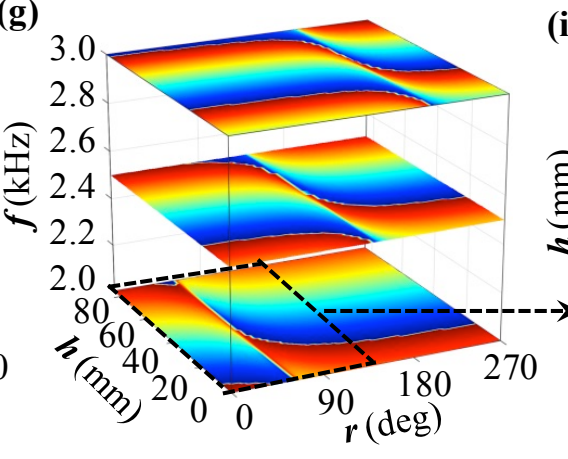

(c)

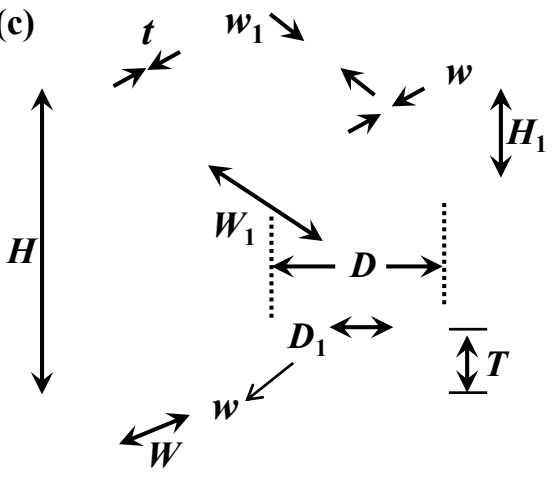

(h) $\quad f=2 \mathrm{kHz}$ Amplitude(a.u.)

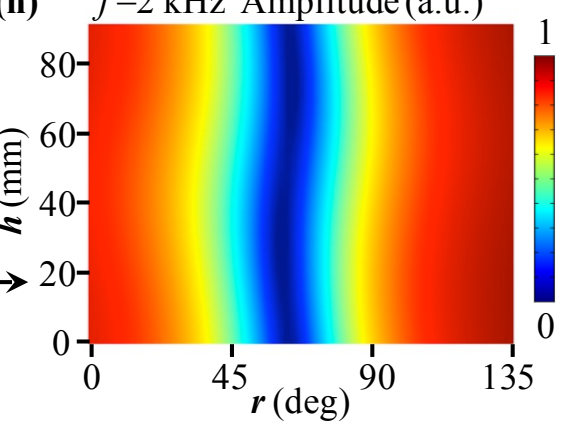

(i)

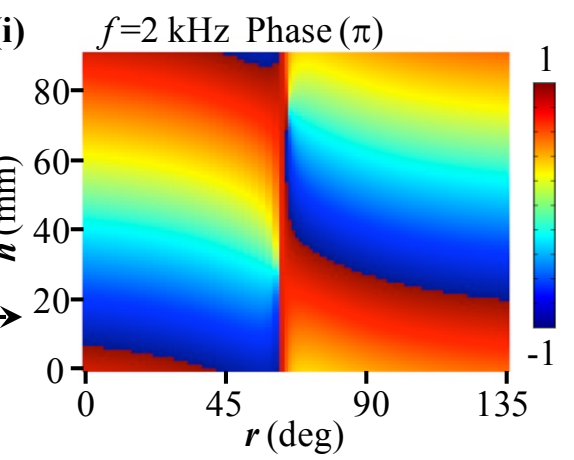

Figure 2. Schematic of the proposed broadband acoustic hologram metasurface with tunable inside 
absorbers. (a) The schematic diagram of the broadband tunable AH. (b) The TLAM unit. (c) The geometric details of the tunable components. $(\mathrm{d}, \mathrm{e})$ The reflected amplitude and phase of the designed unit varying with the height $h=[0,85] \mathrm{mm}$ and the rotational angle $r=[0,270]^{\circ}$ at the frequency domain $f=[2,3] \mathrm{kHz}$. (f, g) The horizontal slices in (d) and (e) at $f=2,2.5,3 \mathrm{kHz}$. (h, i) The local spectrums of the reflected amplitude and phase response varying with $h$ and $r$ at the frequency of $f=2 \mathrm{kHz}$.

As illustrated in figure 2(c), the total height is $H=100 \mathrm{~mm}$, the side length is $W=20 \mathrm{~mm}$, and the wall thickness is $t=0.5 \mathrm{~mm}$. The height of the helical gap is $H_{1}=10 \mathrm{~mm}$ and the side length $W_{1}=19$ $\mathrm{mm}$. The gap width is $w=1 \mathrm{~mm}$ and the length $w_{1}=6 \mathrm{~mm}$. The external and internal diameters of the matched helix are $D=18 \mathrm{~mm}$ and $D_{1}=6 \mathrm{~mm}$, respectively. The blade thickness is the same as the gap width $w$; and the thread lead $T=8 \mathrm{~mm}$. It should be noted that four matched helixes are attached together with a thin sheet and set up a ring gear by distributing grooves on its edge to facilitate the rotation.

To show the broadband tunability, we should first obtain the relation between the reflected amplitude-phase and the tunable parameters in a continuous frequency domain. The thermoviscous acoustics model is employed in the gaps of the TLAM unit with both the viscous friction and thermal diffusion being considered, see Appendix C for details.

Figures 2(d) and 2(e) show the reflected amplitude and phase of the designed unit varying with the height $h=[0,85] \mathrm{mm}$ and the rotational angle $r=[0,270]^{\circ}$ in the frequency domain $f=[2,3] \mathrm{kHz}$, respectively. In order to see the distributions inside the 3D spectrums, the horizontal slices in (d) and 
(e) at $f=2,2.5,3 \mathrm{kHz}$ are shown in figures $2(\mathrm{f})$ and $2(\mathrm{~g})$, respectively. For more clarity, the local spectra are zoomed in to figures2(h) and 2(i) at the frequency of $f=2 \mathrm{kHz}$. It is found that the full modulation of the amplitude and phase can be achieved by using the TLAM unit. It is also noted that the manipulation of the amplitude is nearly decoupled from the parameter $h$, because it is almost constant in the vertical direction. That means we can individually adjust the amplitude by rotating the angle $r$, i.e., $A=A(r)$. However, the phase can be determined by combining two parameters, i.e., $\varphi=\varphi(r, h)$, see figure 2(i). Note that the smallest amplitude appears at $r=61^{\circ}$, where the quasi-perfect absorption [42] occurs with the phase always being around $\pi$, as we mentioned in the last section. Therefore, it is necessary to avoid this position when we extract the tunable parameters according to the desired amplitude and phase.
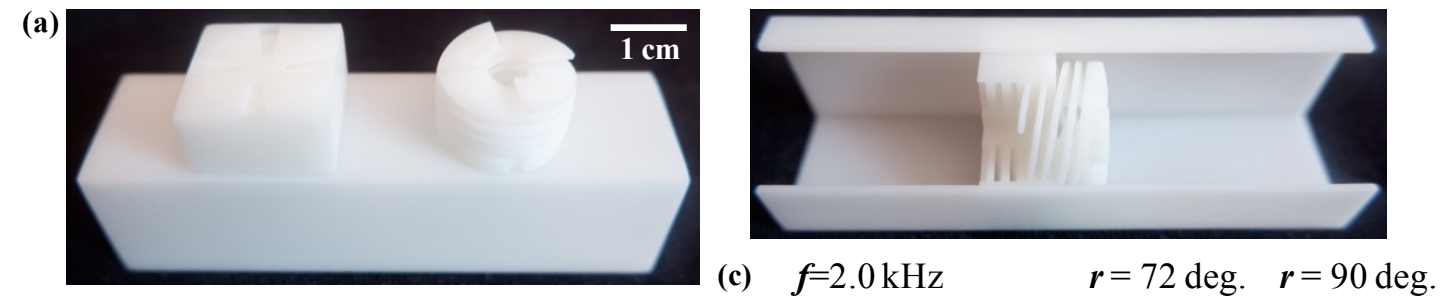

(b)

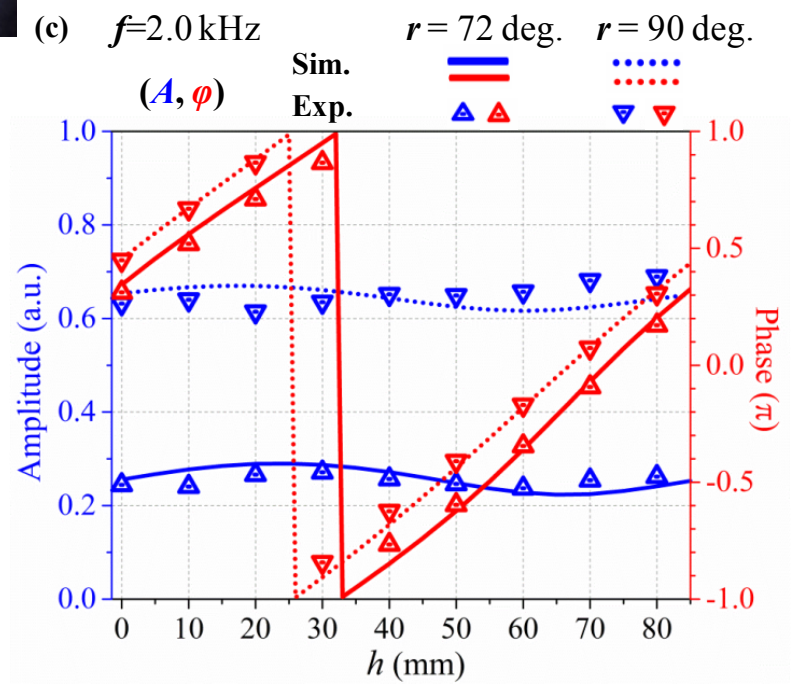


Figure 3. The simulated and measured amplitude and phase varying with the height $h$ at two particular rotational angles $\boldsymbol{r}$ at the frequency of 2 kHz. (a) Photographs of the fabricated TLAM unit. (b) Schematic of the experimental set up. (c) The simulated (Sim.) and experimental (Exp.) results of the amplitude (blue) and phase (red).

The experiment is carried out to evidence the acoustic amplitude and phase tuned by the designed TLAM unit. The components of the TLAM unit made of epoxy resin via 3D printing with enough precision $(0.1 \mathrm{~mm})$ are shown in figure $3(\mathrm{a})$. Figure $3(\mathrm{~b})$ shows the schematic diagram of the experimental setup. We refer to Appendix $\mathrm{C}$ for the details of the devices and the measurement processes. To illustrate the extraction of the tunable parameters from a quantitative perspective, we present the numerical and experimental results of the amplitude and phase varying with the height $h$ at the rotational angles of $r=72^{\circ}$ and $90^{\circ}$ at the frequency of $2 \mathrm{kHz}$ in figure 3(c). The results clearly show that the TLAM unit can provide a full-range phase control $(-\pi \sim+\pi)$ with nearly constant amplitudes being around 0.25 and 0.65 by tailoring $h$, which means that we can first determine the rotational angle $r$ based on the needed amplitude, and then search the corresponding height $h$ according to the required phase in a $2 \pi$ range, eventually achieve the decoupling of the APM. It should be pointed out that if the desired amplitude is very small $(A<0.1)$ or very large $(A>0.95)$, it should be taken as 0.1 or 0.95 .

\section{The fine manipulation of sound fields for broadband acoustic holography}




\subsection{Scattering-free anomalous reflection}

We start with the analysis of anomalous reflection by considering a plane acoustic wave impinging on a gradient AM, as shown in figure 4(a), where $p_{\mathrm{i}}$ and $p_{\mathrm{r}}$ denote the incident and reflected waves; $\theta_{\mathrm{i}}$ is the incident angle; and $\theta_{\mathrm{r}}$ is the desired reflected angle. We suppose that the counterclockwise is positive. The anomalous reflection, as a fundamental function is the basis of many interesting applications $[6,7]$.

(a)

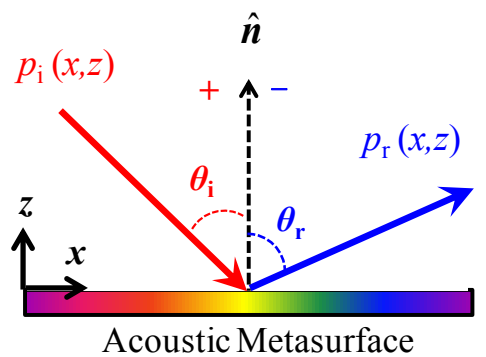

(d)
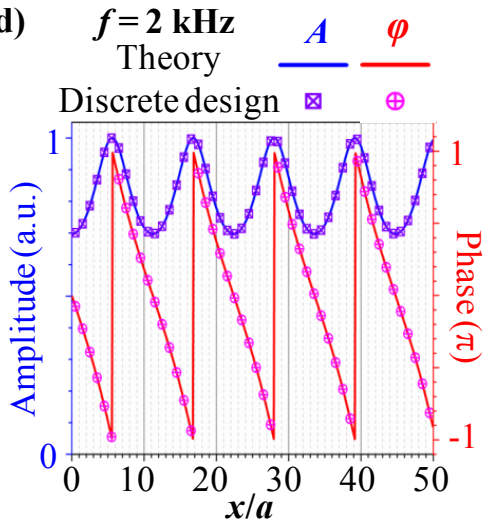

(b)

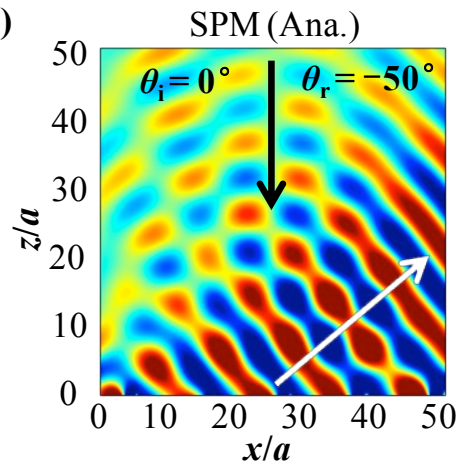

(e)

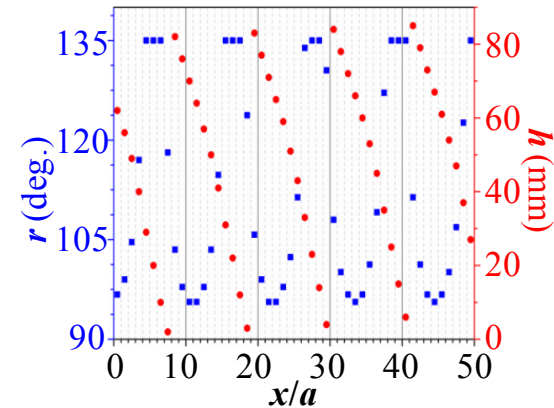

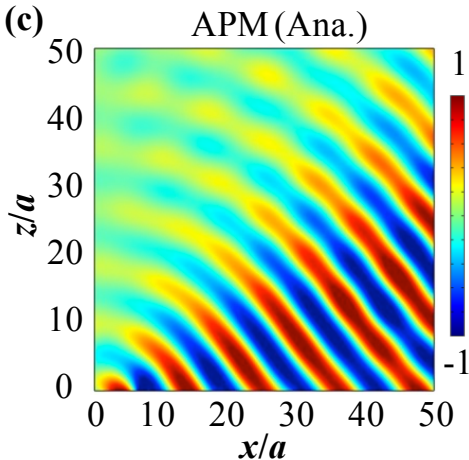

(f)

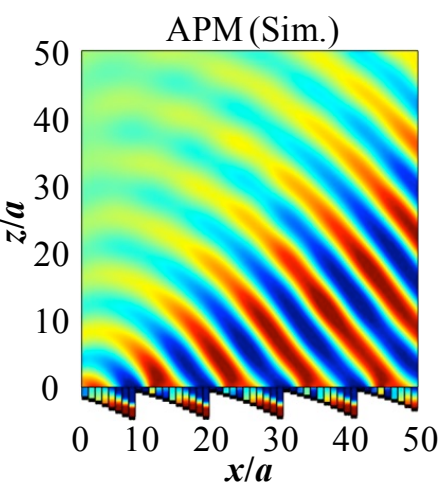

Figure 4. The scattering-free anomalous reflection for fine manipulation of sound fields. (a) Schematic of an AM for a plane wave. (b) The analytical reflected pressure field for $\theta_{\mathrm{i}}=0^{\circ}$ and $\theta_{\mathrm{r}}=-50^{\circ}$ by the single phase-modulation (SPM) method at the frequency of $2 \mathrm{kHz}$. (c) The analytical reflected pressure field by the amplitude-phase-modulation (APM) method. (d) The amplitudes and phases required to reflect the normally incident plane waves into desired direction waves of $\theta_{\mathrm{r}}=-50^{\circ}$ by the APM method. (e) The required tunable 
parameters including the height $h$ and the rotational angle $r$ for each unit of the designed TLAM. (f) The corresponding simulated pressure field by the APM method .

The conventional designs based on the generalized Snell's law are to introduce a linear gradient phase shift $\left[\varphi_{x}=k x\left(\sin \theta_{\mathrm{r}}-\sin \theta_{\mathrm{i}}\right)\right]$ in the artificial AM. That means the AM is characterized by SPM method and the local reflection coefficient with the unit amplitude is $R_{0}=\exp \left(i \varphi_{x}\right)$. The reflection coefficient is related with the surface impedance as $R_{0}=\left(Z_{\mathrm{S}}-Z_{\mathrm{i}}\right) /\left(Z_{\mathrm{S}}+Z_{\mathrm{i}}\right)$, where $Z_{\mathrm{i}}=\rho_{0} c_{0} / \cos \theta_{\mathrm{i}}$ represents the characteristic impedance of the incident wave. Thus, the surface impedance of the AM can be inversely derived as

$$
Z_{\mathrm{S}}^{\mathrm{OPM}}(x)=i \rho_{0} c_{0} \cot \left[k x\left(\sin \theta_{\mathrm{r}}-\sin \theta_{\mathrm{i}}\right)\right] / \cos \theta_{\mathrm{i}} .
$$

We model the AM as an impedance boundary described by Eq. (4) and analyze its reflected field in figure 4(b) for $\theta_{\mathrm{i}}=0^{\circ}$ and $\theta_{\mathrm{r}}=-50^{\circ}$ at the frequency of $2 \mathrm{kHz}$. Clearly, in addition to the desired anomalously reflected wave, some waves along other directions will be excited in this field due to the parasitic diffraction [43]. Then the simple design philosophy by SPM method does not ensure the perfect energy conversion between the incident and reflected plane waves.

Inspired by the leaky-wave antennas [44], undesired diffraction is expected to be suppressed by allowing power absorption in our tunable unit. Then the APM method can be used to design a flat TLAM for scattering-free anomalous reflection [45]. Assuming the time-harmonic factor, the acoustic pressures and the velocity vectors associated with the space pressure fields are 


$$
\begin{aligned}
& p_{\mathrm{i}}(x, z)=p_{0} \exp \left[-i k\left(x \sin \theta_{\mathrm{i}}-z \cos \theta_{\mathrm{i}}\right)\right], \\
& p_{\mathrm{r}}(x, z)=A_{\mathrm{r}} p_{0} \exp \left[-i k\left(x \sin \theta_{\mathrm{r}}+z \cos \theta_{\mathrm{r}}\right)\right], \\
& \vec{v}_{\mathrm{i}}(x, z)=p_{\mathrm{i}}(x, y)\left(\hat{x} \sin \theta_{\mathrm{i}}-\hat{z} \cos \theta_{\mathrm{i}}\right) / \rho_{0} c_{0}, \\
& \vec{v}_{\mathrm{r}}(x, z)=p_{\mathrm{r}}(x, y)\left(\hat{x} \sin \theta_{\mathrm{r}}+\hat{z} \cos \theta_{\mathrm{r}}\right) / \rho_{0} c_{0},
\end{aligned}
$$

where $A_{\mathrm{r}}$ is a constant which relates the amplitude of the reflected pressure field normalized by the incident one. The surface impedance can be expressed as:

$$
Z_{\mathrm{S}}^{\mathrm{APM}}(x)=\frac{p_{\mathrm{tot}}(x)}{-\hat{n} \cdot \vec{v}_{\mathrm{tot}}(x)}=\frac{\rho_{0} c_{0}\left[1+A_{\mathrm{r}} \exp \left(\mathrm{i} \varphi_{x}\right)\right]}{\cos \theta_{\mathrm{i}}-A_{\mathrm{r}} \cos \theta_{\mathrm{r}} \exp \left(\mathrm{i} \varphi_{x}\right)}
$$

where $\hat{n}$ is the unit vector along the normal direction; and $p_{\text {tot }}=p_{\mathrm{i}}+p_{\mathrm{r}}$ and $\vec{v}_{\text {tot }}=\vec{v}_{\mathrm{i}}+\vec{v}_{\mathrm{r}}$ denote the total acoustic pressure and velocity, respectively. The real part of the surface impedance will become negative (gain) when $\theta_{\mathrm{i}}>\left|\theta_{\mathrm{r}}\right|$, meaning that additional energy has to be introduced in the system [45]. It is not suitable for the passive loss structure we designed.

In order to eliminate parasitic scattering, there must be no power flowing in the unwanted direction from any point on the surface. Then, the local emerging power at the surface can be expressed in terms of the normal component of the intensity vector

$$
\begin{aligned}
P_{\mathrm{n}} & =\left.\hat{n} \cdot \frac{1}{2} \operatorname{Re}\left[p_{\mathrm{tot}} \vec{v}_{\mathrm{tot}}^{*}\right]\right|_{y \rightarrow 0^{+}} \\
& =\frac{p_{0}^{2}}{2 \rho_{0} c_{0}}\left[-\cos \theta_{\mathrm{i}}+A_{\mathrm{r}}\left(\cos \theta_{\mathrm{r}}-\cos \theta_{\mathrm{i}}\right) \cos \varphi_{x}+A_{\mathrm{r}}^{2} \cos \theta_{\mathrm{r}}\right],
\end{aligned}
$$


where "*" represents the complex conjugate. Enforcing $P_{\mathrm{n}} \leqslant 0$ for all $x$ points of the TLAM, it is found that the maximum value of $A_{\mathrm{r}}$ is $\min \left[1, \cos \theta_{\mathrm{i}} / \cos \theta_{\mathrm{r}}\right]$. The conditions $\theta_{\mathrm{i}} \leq\left|\theta_{\mathrm{r}}\right|$ and $A_{\mathrm{r}}=1$ can satisfy this situation. Thus, the surface impedance can be written as:

$$
Z_{\mathrm{S}}^{\mathrm{APM}}(x)=\frac{\rho_{0} c_{0}\left[\exp \left(-i k x \sin \theta_{\mathrm{i}}\right)+\exp \left(-i k x \sin \theta_{\mathrm{r}}\right)\right]}{\cos \theta_{\mathrm{i}} \exp \left(-i k x \sin \theta_{\mathrm{i}}\right)-\cos \theta_{\mathrm{r}} \exp \left(-i k x \sin \theta_{\mathrm{r}}\right)}
$$

Figure. 4(c) shows the analytical pressure field with modeling the TLAM as an impedance boundary described by Eq. (8), corresponding to the previous case in figure $4(\mathrm{~b})$, for $\theta_{\mathrm{i}}=0^{\circ}$ and $\theta_{\mathrm{r}}=-50^{\circ}$ at the frequency of $2 \mathrm{kHz}$. By comparing figure 4(b) and (c), it clearly appears that the reflected wave from the TLAM propagates only along the desired direction without parasitic waves. These results confirm the excellent performance of the anomalous reflection by APM method.

The corresponding local reflection coefficient are

$$
R_{a}(x)=\frac{Z_{\mathrm{S}}^{\mathrm{APM}}-Z_{i}}{Z_{\mathrm{S}}^{\mathrm{APM}}+Z_{i}}=\frac{\left(\cos \theta_{\mathrm{i}}+\cos \theta_{\mathrm{r}}\right) \exp \left(-i k x \sin \theta_{\mathrm{r}}\right)}{2 \cos \theta_{\mathrm{i}} \exp \left(-i k x \sin \theta_{\mathrm{i}}\right)+\left(\cos \theta_{\mathrm{i}}-\cos \theta_{\mathrm{r}}\right) \exp \left(-i k x \sin \theta_{\mathrm{r}}\right)}
$$

To show the performance of the designed TLAM structure, full-wave simulation of the reflected field will be carried out with COMSOL in the same case for overall comparisons. Figure 4(d) presents the amplitude $\left[A=\operatorname{abs}\left(R_{a}\right)\right]$ and phase $\left[\varphi=\arg \left(R_{a}\right)\right]$ required to achieve the scattering-free anomalous reflection by the APM method. The discretized amplitudes and phases of the 50 units are marked by squares and circles, respectively. The associated $h$ and $r$ for each unit can be obtained from figure 2 and plotted by the scattered dots in figure 4(e). By employing these parameters, the corresponding 
reflected acoustic field is simulated and illustrated in figure 4(f). The result indicates that the designed TLAM by APM method can perfectly realize the scattering-free function to reflect the waves in the desired direction, which is as good as the analysis in figure 4(c) and significantly better than the one by SPM method in figure 4(b).

\subsection{Scattering-free multi-plane acoustic hologram images}

(a)

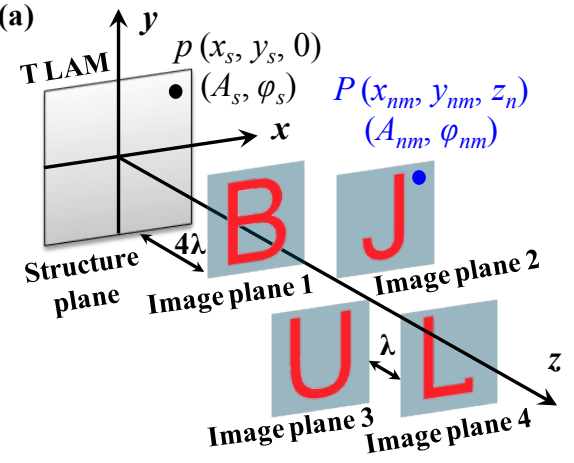

(d)

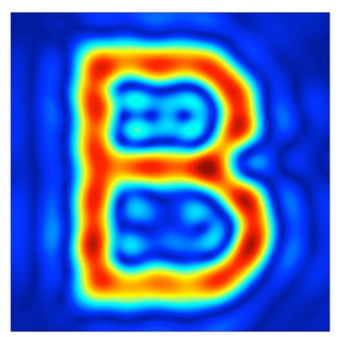

Image plane 1

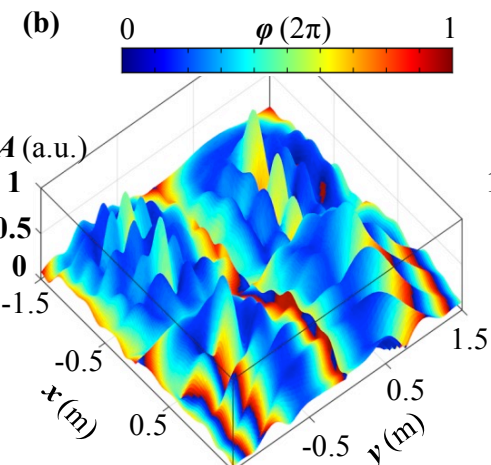

$1.5-1.5$

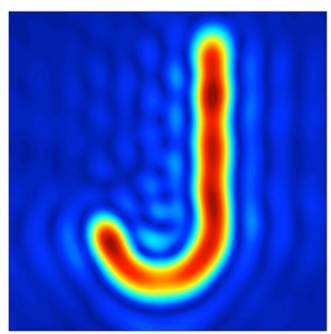

Image plane 2

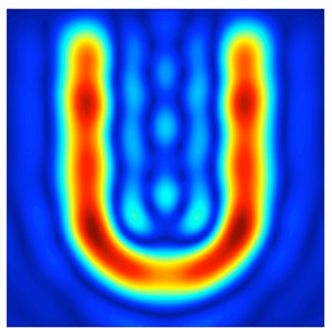

Image plane 3

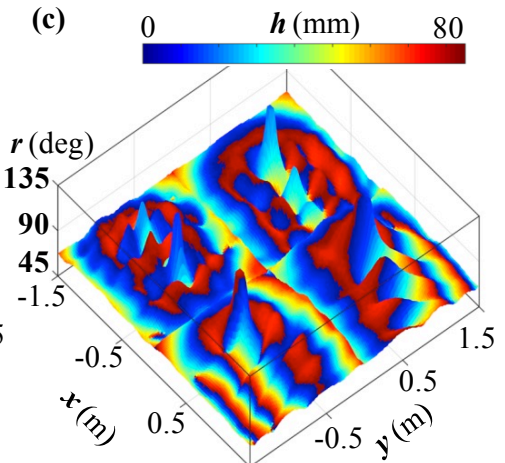

$1.5^{-}-1.5$

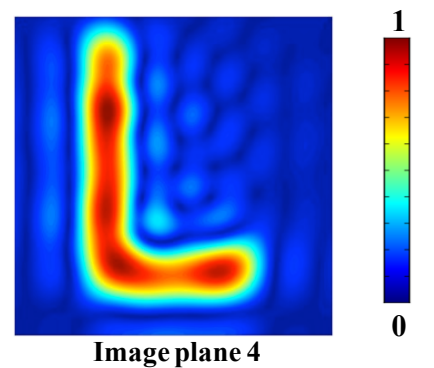

Figure 5. The scattering-free multi-plane acoustic hologram images. (a) Schematic of the predesigned images for a multi-plane $\mathrm{AH}$ (letters $\mathrm{B}, \mathrm{J}, \mathrm{U}$ and $\mathrm{L}$ at different positions from $4 \lambda$ to $7 \lambda$ by step of $\lambda$ at the frequency of 2 $\mathrm{kHz}$ ). (b) The required amplitude and phase profiles on the hologram plane for projecting these four images at multiple planes. (c) The corresponding needed parameters (i.e., the varying height $h$ and the rotational angle $r$ ) for the designed TLAM structure. (d) The scattering intensity field of the holographic images by the APM method.

Due to the scattering-free property of the TLAM, we show the fine control of acoustic waves by 
hologram projection of multi-plane images. The general principle for acoustic hologram reconstruction at multiple planes is depicted in figure 5(a).

The phase and amplitude profiles at the structure plane are calculated using time-reversal acoustics $[12,20]$. First, the $N$ th predesigned image is discretized into $M^{2}$ pixels, and then each "image pixel" is defined as $p_{n \mathrm{~m}}=A_{n m} \exp \left(i \varphi_{n m}\right)$, where $A_{n m}$ and $\varphi_{n m}$ are the amplitude and initial phase of the point $\left(x_{n m}, y_{n m}, z_{n}\right)$ at the image plane. Therefore, the acoustic pressure with amplitude $A_{s}$ and phase $\varphi_{s}$ on the structure plane of the TLAM can be calculated by superposing the wave components from those image pixels, as follows

$$
p_{s}\left(x_{s}, y_{s}, 0\right)=A_{s} \exp \left(i \varphi_{s}\right)=\sum_{n=1}^{N} \sum_{m=1}^{M^{2}} \frac{A_{n m}}{d_{s n m}} \exp \left[i\left(k d_{s n m}+\varphi_{n m}\right)\right]
$$

where $d_{s n m}=\sqrt{\left(x_{s}-x_{n m}\right)^{2}+\left(y_{s}-y_{n m}\right)^{2}+z_{n}^{2}}$ is the distance between the image pixel and the TLAM unit with a coordinate of $\left(x_{s}, y_{s}, 0\right)$ at the structure plane. Then, the holographic image will agree with the predesigned one due to the time-reversal symmetry, which can be characterized by

$$
P(x, y, z)=\sum_{s=1}^{S} \frac{A_{s}}{d_{s}} \exp \left[-i\left(k d_{s}-\varphi_{s}\right)\right]
$$

where $S$ is the total number of the structure pixels, i.e., the number of the units. The distance between the spatial point and the structure pixel of the TLAM is $d_{s}=\sqrt{\left(x-x_{s}\right)^{2}+\left(y-y_{s}\right)^{2}+z^{2}}$. Thus, it is important to introduce the simultaneous modulation of the amplitude and phase, which is straightforward by controlling $\left(A_{s}, \varphi_{s}\right)$ at the structure plane to generate $\left(A_{n m}, \varphi_{n m}\right)$ at the multiple 
image planes. Note that the positions of the images should avoid superposition in the reflected direction along $z$-axis, because the scattering-free reflection is valid for deviating from the propagation direction.

Consider the case that the reflective direction is normal to the structure plane, as shown in figure 5(a), where the holographic images are predesigned to be letters $\mathrm{B}, \mathrm{J}, \mathrm{U}$, and $\mathrm{L}$ at four different planes $(N=4)$. They are spaced from $4 \lambda$ to $7 \lambda$ by a step of $\lambda$ away from the structure plane at the frequency of $2 \mathrm{kHz}$. Each target image comprises $79 \times 79$ image pixels $(M=79)$. The size of the effective holographic regions at each image plane is $1.5 \times 1.5 \mathrm{~m}^{2}$ with locations at different quadrants in their $x-y$ plane. We record amplitude and phase distributions in figure 5(b), and then extract the corresponding heights and the rotational angles in figure $5(\mathrm{c})$ for each image with $75 \times 75$ units. The number of the units $(S)$ is chosen by comprehensively considering the complexity of the predesigned images within next section, because we expect to implement arbitrary images in broadband by using the same designed TLAM due to its reconfigurability. Then, figure 5(d) illustrates the simulated distributions of acoustic intensity by the APM method. The results agree well with the predesigned ones for the four image planes, which demonstrates the excellent multi-plane holographic imaging of the TLAM.

\subsection{High-fidelity acoustic hologram in broadband}

Yet the success of our proposed TLAM in generating multi-plane acoustic holograms is by no means 
a limit of its potential application. The engineering of the tunable loss in AMs is indeed significant for controlling sound fields at will and may have applications in various scenarios [46, 47]. As typical examples, we then further systematically investigate the projections of the arbitrary images in a broadband range.

(a)
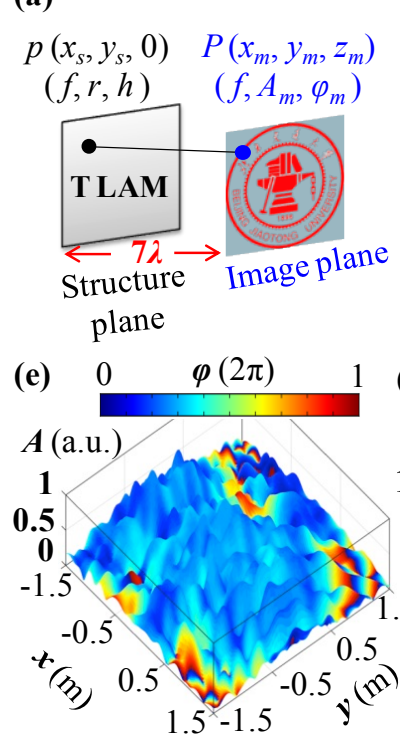

(i) $f=3.0 \mathrm{kHz} \quad R_{\mathrm{CL}}=0.875$

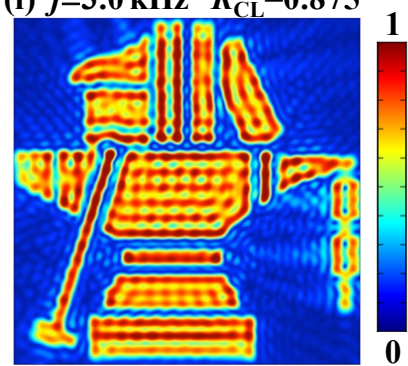

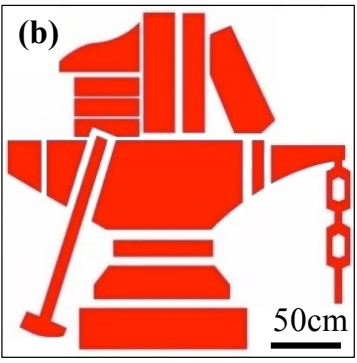
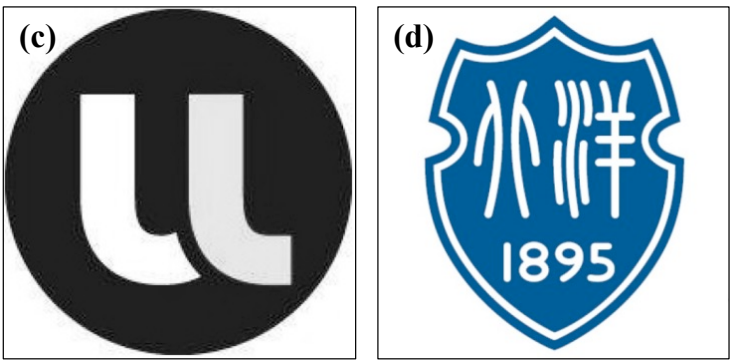

(g) $f=2.0 \mathrm{kHz} \quad R_{\mathrm{CL}}=0.811$ (h) $f=2.5 \mathrm{kHz} R_{\mathrm{CL}}=0.851$
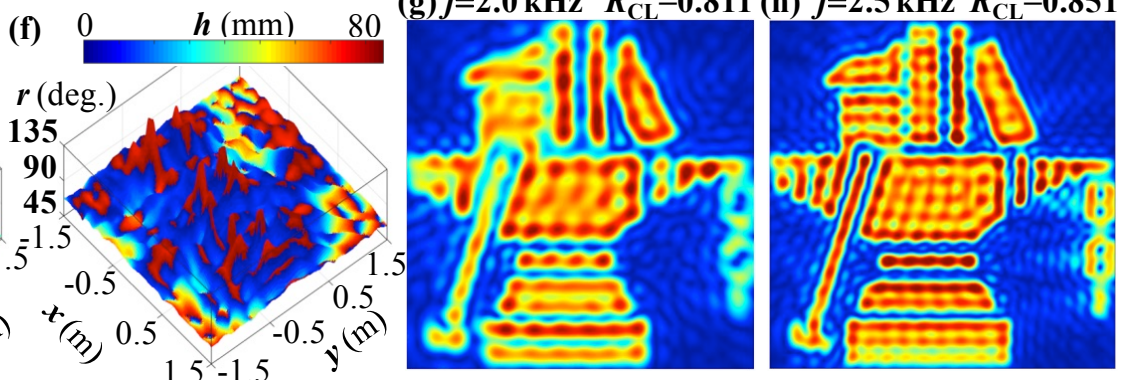

$\begin{array}{ll}1.5 & -1.5 \\ \text { (j) } f=3.0 \mathrm{kHz} & R_{\mathrm{CL}}=0.912\end{array}$

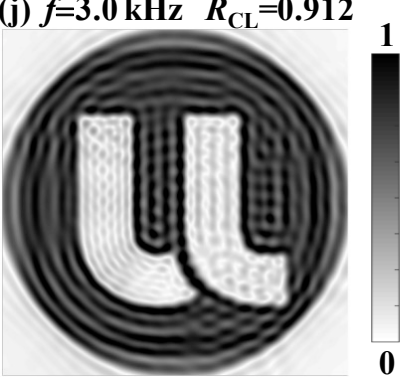

(k) $f=3.0 \mathrm{kHz} \quad R_{\mathrm{CL}}=0.767$

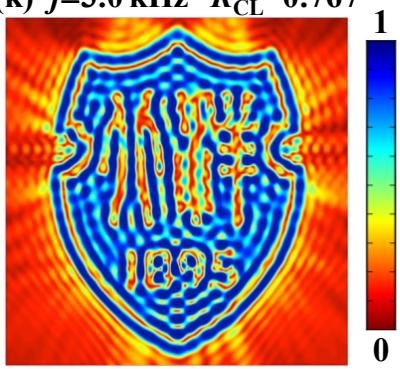

Figure 6. The broadband high-fidelity images of the acoustic hologram. (a) Schematic diagram of hologram reconstruction in the distance of $7 \lambda$ at different frequencies. The target images about three university badges with complex pixel distributions are shown in (b), (c) and (d), respectively. The required amplitude and phase profiles on the hologram plane (e) and the needed tunable parameters of the designed TLAM (f) are calculated and exported to project the image (b) at the frequency of $2 \mathrm{kHz}$. (g) The corresponding simulated result of the holographic image (b). (h) and (i) are the holographic image (b) at the frequencies of 2.5 and $3.0 \mathrm{kHz}$, respectively. The holographic 
images (c) and (d) are shown in (j) and (k) at the frequency of $3.0 \mathrm{kHz}$, respectively.

Figure 6(a) shows the schematic of hologram reconstructions at different frequencies. The holographic images are located at a distance of $7 \lambda$ from the structure surface of the TLAM. Then, the target images about three university badges with relatively complex pixel distributions $(M=201)$ are shown in figures $6(\mathrm{~b}), 6(\mathrm{c})$ and $6(\mathrm{~d})$, respectively. To project the image figure $6(\mathrm{~b})$ at the frequency of $2 \mathrm{kHz}$, the required amplitude-phase profile on the structure plane and the corresponding tunable parameters of the designed TLAM are calculated and exported in figures 6(e) and 6(f). The simulated result of the holographic image is displayed in figure $6(\mathrm{~g})$. In order to exhibit the broadband tunability, figures $6(\mathrm{~h})$ and $6(\mathrm{i})$ present the same image at the frequencies of 2.5 and $3.0 \mathrm{kHz}$, respectively. It is shown that the imaging performances are acceptable intuitively although there is a slight difference in detail at different frequencies. We also reveal the different predesigned images (i.e., figures $6(\mathrm{c})$ and $6(\mathrm{~d})$ ) in figures $6(\mathrm{j})$ and $6(\mathrm{k})$ at the same frequency of $3 \mathrm{kHz}$. The results demonstrate the effectiveness and flexibility of our TLAM design in AH reconstruction. The corresponding required parameter profiles are given clearly in Appendix A for projecting figures 6(h), 6(i), 6(j) and 6(k).

For a more quantitative evaluation of the $\mathrm{AH}$ quality, we introduce the following correlation coefficient [48] to calculate the similarity between the generated holographic image and the original predesigned one: 


$$
R_{\mathrm{CL}}=\frac{\sum_{u=1}^{M} \sum_{v=1}^{M}\left(A_{u v}-\bar{A}\right)\left(B_{u v}-\bar{B}\right)}{\sqrt{\left[\sum_{u=1}^{M} \sum_{v=1}^{M}\left(A_{u v}-\bar{A}\right)^{2}\right]\left[\sum_{u=1}^{M} \sum_{v=1}^{M}\left(B_{u v}-\bar{B}\right)^{2}\right]}},
$$

where $A$ and $B$ are the data matrices of the two images; $\bar{A}$ and $\bar{B}$ are the mean values of the elements in these two matrices, respectively. Note that the contrast of the data matrices requires the images to be shown in grayscale without transparency, see Appendix B. The calculated correlations for figure $6(\mathrm{~b})$ are $0.811,0.851,0.875$ at the frequencies of $2.0,2.5$ and $3.0 \mathrm{kHz}$, respectively, which indicates that the qualities of the holograms are enhanced with the frequency increasing. This can be explained by the fact that each frequency can be designed as the target one in the present broadband range due to the structural tunability, and then the higher frequencies are equivalent to the increased resolution of the diffraction effect. In striking contrast, the performances of the traditional fixed AMs $[6,12,49]$ will be weakened with deviating from the designed frequency even for high frequencies. In addition, the comparisons for $R_{\mathrm{CL}}$ at the frequency of $3 \mathrm{kHz}$ illustrate that the quality of the $\mathrm{AH}$ is also determined by the complexity of the predesigned images. Then, all of the holographic images have a good projection quality with the correlation coefficient greater than 0.76 . The results reveal that our designed TLAM based on APM method has a relatively high-fidelity AH performance in a continuous frequency range.

\section{Conclusion}


In summary, we have proposed a systematic design of the TLAM by engineering the intrinsic losses in broadband based on the matched helical mechanism. Unlike the previous reported schemes, we designed a tunable lossy systems without the foamed materials to independently modulate the reflected amplitude and phase, and discussed the fundamental physics behind their generations. The excellent performance of the TLAM is demonstrated by the scattering-free anomalous reflection as an important basic function, and then illustrated by the multi-plane acoustic holograms and the broadband holographic images. The variety of acoustic projecting applications can be realized by the flexible reconfiguration of one designed metasurface. Therefore, the developed tunable mechanism can significantly increase the degrees of freedom for the classical AMs, which could open up an flexible way to deal with the general idea of multiple acoustic communications, broadband particle manipulations, and stereo sound-field reconstructions, etc.

\section{Acknowledgment}

This work is supported by the National Natural Science Foundation of China (Nos. 11872101 and 11991031), by la Région Grand Est, and by the Institut CARNOT ICEEL. The first author is grateful for the support of the National Natural Science Foundation of China (No. 11502123), the Natural 
Science Foundation of Inner Mongolia Autonomous Region of China (No. 2015JQ01), and China Scholarship Council (CSC No. 201807090043). 


\section{Appendix A: Distributions of the amplitude-phase and tunable parameters for}

\section{the designed broadband AH images}

For convenience and clarity, the following figure A1 shows the distributions of the amplitudes and phases as well as the tunable two parameters (i.e., the varying height $h$ and rotational degree $r$ ) for the AH images in figure 6 at different frequencies. As checked and referenced values, these data may be used to tune the functions of the designed TLAM.

(a) $f=2.5 \mathrm{kHz}$ Fig. $6(\mathrm{~h})$

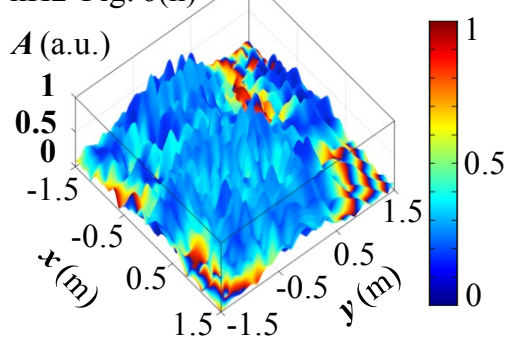

(b) $f=3.0 \mathrm{kHz}$ Fig. 6(i)

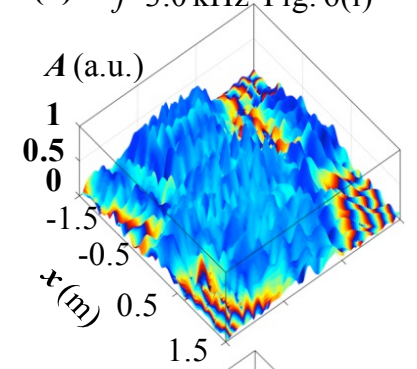

(c) $f=3.0 \mathrm{kHz}$ Fig. $6(\mathrm{j})$

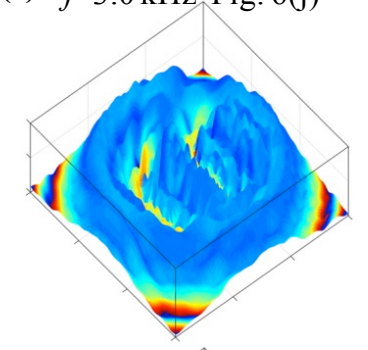

(d) $f=3.0 \mathrm{kHz}$ Fig. $6(\mathrm{k})$
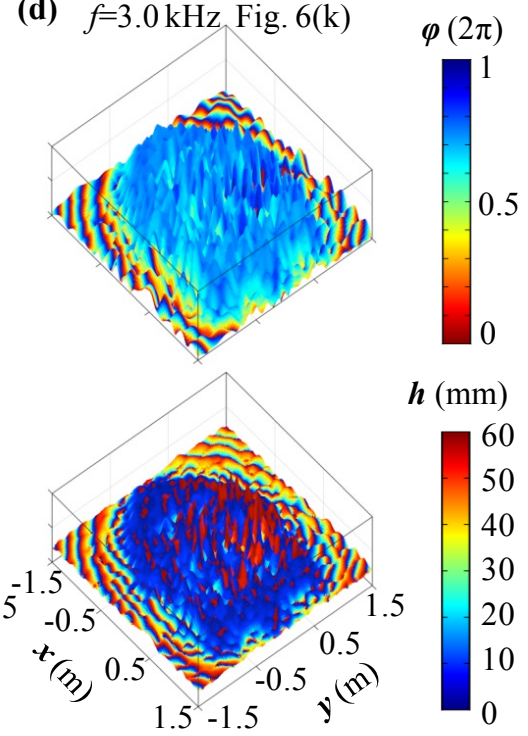

Figure A1. The required amplitude and phase profiles on the structure plane and the corresponding needed tunable parameters of the designed TLAM for projecting the images of figure $6(\mathrm{~h})$ at the frequency of $2.5 \mathrm{kHz}$ and figures 6(i), 6(j) and 6(k) at the frequency of $3.0 \mathrm{kHz}$ are shown in (a), (b), (c) and (d), respectively. 


\section{Appendix B: Quantitative evaluation of the quality of acoustic holograms}

The correlation coefficients are evaluated between the predesigned images and the holographic ones rendered in grayscale, as shown in figure B1.

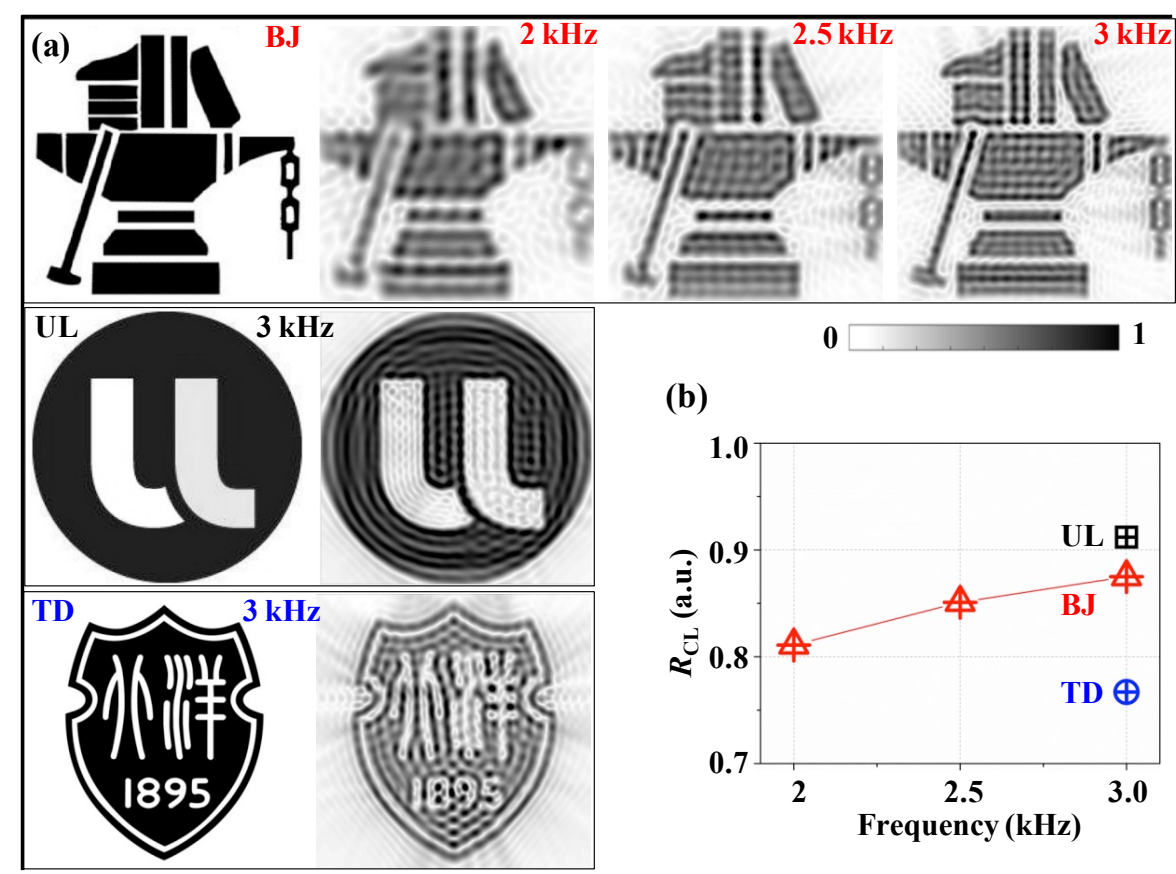

Figure B1. (a) The original predesigned images and the generated holographic ones to be shown in grayscale without transparency. (b) The corresponding correlation coefficients for images at different frequencies.

\section{Appendix C: Numerical simulation and experimental measurement}

The numerical results are calculated in the range from 2 to $3 \mathrm{kHz}$ by using the Acoustics-Thermoviscous Acoustics Interaction Module in COMSOL Multiphysics 5.5 software. The thermoviscous acoustic module is employed in the regions of helical gaps. This interface solves the following continuity equation, momentum equation (Navier-Stokes equation), and energy equation [34], respectively, 


$$
\begin{aligned}
& i \omega \rho+\nabla \cdot\left(\rho_{0} \boldsymbol{u}\right)=0, \\
& i \omega \rho_{0} \boldsymbol{u}=\nabla \cdot\left[-p \boldsymbol{I}+\eta\left[\nabla \boldsymbol{u}+(\nabla \boldsymbol{u})^{T}\right]\right]-(2 \eta / 3)(\nabla \cdot \boldsymbol{u}) \boldsymbol{I}, \\
& \rho_{0} C_{p}\left(i \omega T+\boldsymbol{u} \cdot \nabla T_{0}\right)-\alpha_{p} T_{0}\left(i \omega p+\boldsymbol{u} \cdot \nabla p_{0}\right)=\nabla \cdot(K \nabla T),
\end{aligned}
$$

where $\eta=18.1 \mu \mathrm{Pa} \cdot \mathrm{s}$ is the dynamic viscosity and describes losses due to shear friction $[27,46]$. The thermal conductivity $K=0.0257 \mathrm{~W} /(\mathrm{m} \cdot \mathrm{K})$ is a basic property of the air in the isotropic ray acoustics.

The acoustic intensity vector $\boldsymbol{I}$ is defined as the time average of the instantaneous rate of energy transfer per unit area. The sound velocity $c_{0}=343 \mathrm{~m} / \mathrm{s}$ and the density $\rho_{0}=1.21 \mathrm{~kg} / \mathrm{m}^{3}$ are the default values at the equilibrium pressure $p_{0}=1$ atm and temperature $T_{0}=293.15 \mathrm{~K}$ (that is, $20^{\circ} \mathrm{C}$ ). Then, the pressure $p$, temperature $T$ and velocity $\boldsymbol{u}$ are the dependent variables. The density variation can be expressed as

$$
\begin{aligned}
& \rho=\rho_{0}\left(\beta_{\mathrm{T}} p-\alpha_{p} T\right), \quad \alpha_{p}=\sqrt{C_{p}(\gamma-1) / T_{0}} / c_{0}, \\
& \beta_{\mathrm{T}}=\gamma /\left(\rho_{0} c_{0}^{2}\right), \quad \delta=0.22[\mathrm{~mm}] \sqrt{100[\mathrm{~Hz}] / f},
\end{aligned}
$$

where $C_{p}=1005.4 \mathrm{~J} /(\mathrm{kg} \cdot \mathrm{K})$ is the heat capacity; $\gamma=1.4$ is the dimensionless ratio of specific heats. All the structures are considered as ideal rigid walls. Non-slip and isothermal conditions are imposed on the solid boundaries. The pressure acoustic module is used for the incident and reflected areas. The acoustic-thermoviscous coupling boundary is taken into account. To guarantee calculation precision, the boundary layer properties with thickness $\delta$ of the mesh are set on the inner surfaces of the helical gaps. 


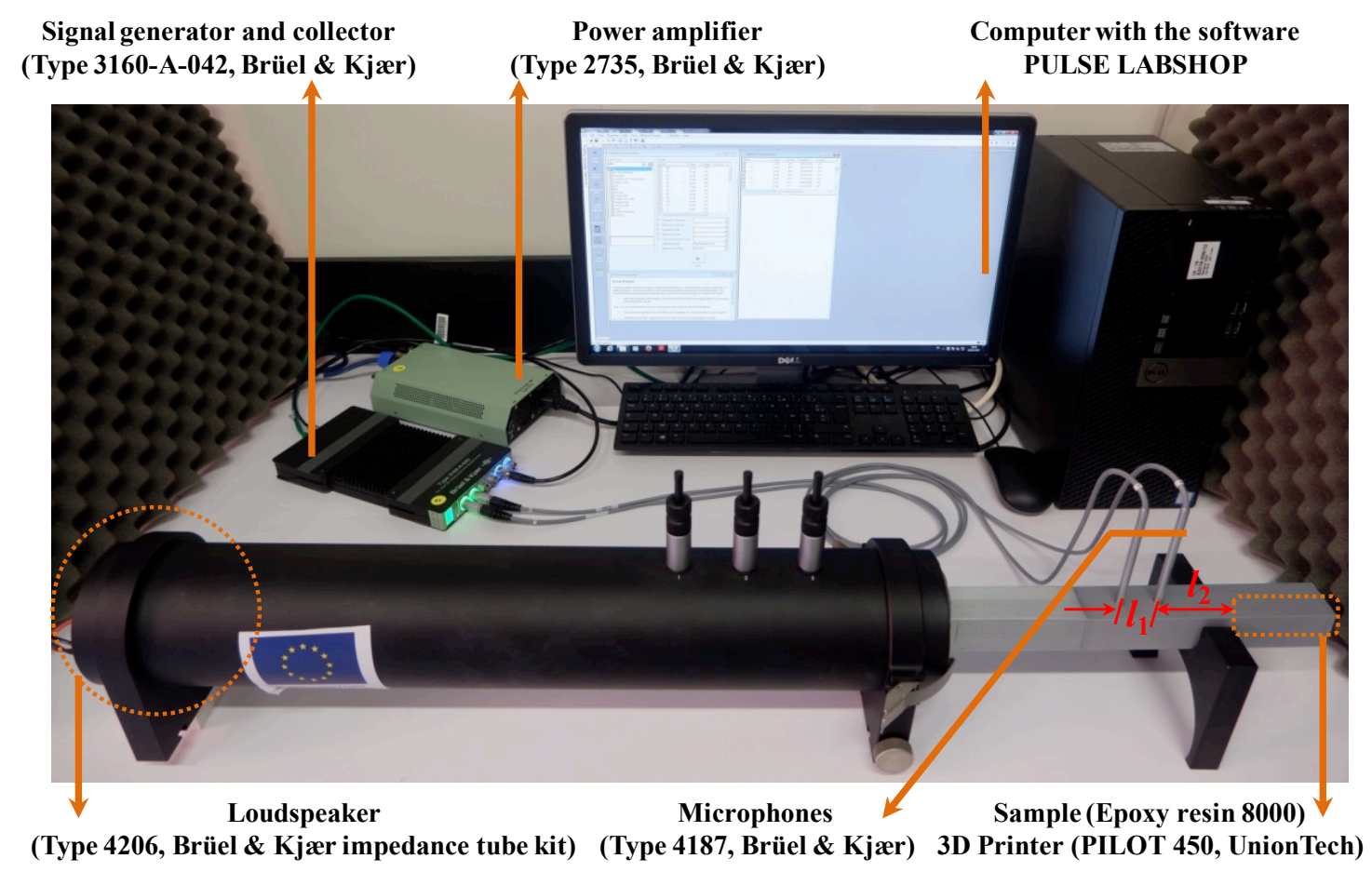

Figure C1. Experimental measurement setup.

As shown in figure $\mathrm{C} 1$, the experimental setup consists of a rectangular waveguide connected with an impedance tube kit (Type 4206, Brüel \& Kjær). The connecting part is $8 \mathrm{~cm}$ in length and is surrounded with the porous foam. The rectangular tube is $38 \mathrm{~cm}$ in total length and has a square cross section with each internal side of $2 \mathrm{~cm}$. Its wall thickness is $1 \mathrm{~cm}$. Two $1 / 4$-inch microphones (Type 4187, Brüel \& Kjær) are situated at designated positions with distance of $l_{1}=3 \mathrm{~cm}$ to sense local pressure. The distance between the second microphone and the unit sample is $l_{2}=6 \mathrm{~cm}$. A 3D printer (PILOT 450, UnionTech) is used to fabricate the components of the TLAM unit with epoxy resin.

Regarding the measurement process, we first use a computer with the software PULSE LABSHOP 
to generate a continuous sound wave signal with a bandwidth of $3.2 \mathrm{kHz}$. The signal is modulated by a power amplifier module (Type 2735, Brüel \& Kjær) and then input to the loudspeaker in the impedance tube. The microphone signals are collected by a LAN-XI data-acquisition hardware (Type 3160-A-042, Brüel \& Kjær). Based on the transfer function method [12, 27], the reflected factor can be obtained as follows

$$
R e=A \exp (i \varphi)=\frac{P_{12}-\exp \left(-i k_{0} l_{1}\right)}{\exp \left(i k_{0} l_{1}\right)-P_{12}} \exp \left[2 i k_{0}\left(l_{1}+l_{2}\right)\right]
$$

where $P_{12}=P_{2} / P_{1}$ is the transfer function between the acoustic pressure measured by the microphones. Then, the reflected amplitude and phase can be directly calculated from $A=\operatorname{abs}(R e)$ and $\phi=\arg (R e)$ by the Brüel \& Kjær PULSE Multi-analyzer system.

Note that the small discrepancies between the simulated and measured results in Fig. 3(c) may be caused by several factors. Since the 3D printed rectangular waveguide cannot guarantee perfect sound insulation, when $h$ is small, the internal absorber is located at the end of the channel, which may cause a slight sound leakage. Inevitably, there exist some tuning errors in operation and the machining errors in fabrication. These errors can affect the resonance effects and may enhance or weaken the sound absorption, resulting in the small discrepancies of the reflected amplitude. In addition, the phase mainly depends on the varying height $h$ (the wide channel length) with low error sensitivity, then its measurement accuracy is higher than the amplitude. All the results from the simulations and experiments are in good agreement. 


\section{Appendix D: The conceptual design of a programmable acoustic holography by}

\section{using the real-time TLAM}

Real-time steering of acoustic waves in a continuous frequency range is one of the key functions required for acoustic programmable holograms. Although the controllable images have been demonstrated based on the designed TLAM, they still have a limitation in dynamic modulation of the acoustic projection, which is a crucial feature for real-world applications in a more smart and convenient way.

\begin{tabular}{|c|c|c|}
\hline \multicolumn{3}{|c|}{ 1. Acoustic holographic image (img.) is predesigned at a certain frequency (freq.). } \\
\hline img. & & freq. \\
\hline \multicolumn{3}{|c|}{ 2. Image analysis module obtains the pixel information. } \\
\hline$\| p_{n \mathrm{~m}}=A_{n m} \exp \left(i \varphi_{n m}\right)$ & & \\
\hline \multicolumn{3}{|l|}{$\begin{array}{l}\text { 3. Image processing module yields the required } \\
\text { amplitude and phase based on Eqs. (10) and (11). }\end{array}$} \\
\hline$\left(A_{s}, \varphi_{s}\right)$ & & \\
\hline \multicolumn{3}{|l|}{$\begin{array}{l}\text { 4. Interpolation calculator acquires the needed } \\
\text { angle and height based on the data in Figure } 2 \text {. }\end{array}$} \\
\hline$\|(r, h)$ & & 6 The system achieves the \\
\hline $\begin{array}{l}\text { 5. Mechanical control system adjusts the } \\
\text { individual unit by a rotating and stepping motor. }\end{array}$ & $\stackrel{\text { Motor }}{\rightleftharpoons}$ & $\begin{array}{l}\text { acoustic hologram, then resets } \\
\text { the programmable TLAM. }\end{array}$ \\
\hline
\end{tabular}

Figure D1. A schematic representation of real-time tunable mechanisms for the programmable acoustic holography.

The illustration at the bottom right shows the structure connective components of the mechanical control system.

Here, we propose a conceptual design of a programmable automatic control system by using our TLAM units, as shown in figure D1. When a holographic image is predesigned to be projected at a 
certain frequency, the image analysis module can obtain the pixel information $\left[p_{n \mathrm{~m}}=A_{n m} \exp \left(i \varphi_{n m}\right)\right]$, then the image processing module yields the required amplitude $A_{s}$ and phase $\varphi_{s}$ based on Eqs. (10) and (11). We can set an interpolation calculator based on all the data in figure 2 to get the necessary angle $r$ and height $h$. The angle and height of each unit can be easily controlled by using a robust rotating and stepping motor. The mechanical control system is the key to achieve the automatic regulation. Its partial details are shown in the illustration at the bottom right. The proposed tunable absorber (white block), 3D printed connector (yellow gear) and a small motor (metallic grey) are assembled into an individually mechanical unit to fabricate a continuously and dynamically tunable system. On the other side of the system, the source receives the frequency information and emits the sound signal. Then the developed tunable system can achieve the acoustic images, and be reset again to program for controlling another designed hologram. This merit of the conceptual design lays the foundation for automatically manipulating acoustic projection using our TLAM structure. 


\section{References}

[1] Zhang J., Tian Y., Cheng Y. and Liu X. 2020 Acoustic holography using composite metasurfaces Appl. Phys. Lett. 116030501

[2] Hertzberg Y. and Navon G. 2011 Bypassing absorbing objects in focused ultrasound using computer generated holographic technique Med. Phys. 386407

[3] Marzo A., Seah S. A., Drinkwater B. W., Sahoo D. R., Long B. and Subramanian S. 2015 Holographic acoustic elements for manipulation of levitated objects Nat. Commun. 68661

[4] Marzo A. and Drinkwater B. W. 2019 Holographic acoustic tweezers Proc. Natl. Acad. Sci. USA 11684

[5] Wang Y. F., Wang Y. Z., Wu B., Chen W. and Wang Y. S. 2020 Tunable and Active Phononic Crystals and Metamaterials Appl. Mech. Rev. 72040801

[6] Assouar B., Liang B., Wu Y., Li Y., Cheng J. C. and Jing Y. 2018 Acoustic metasurfaces Nat. Rev. Mater. 3460

[7] Yu N., Genevet P., Kats M. A., Aieta F., Tetienne J. P., Capasso F. and Gaburro Z. 2011 Light propagation with phase discontinuities: generalized laws of reflection and refraction Science 334333 [8] Cao L., Yang Z., Xu Y., Fan S. W., Zhu Y., Chen Z., Vincent B. and Assouar B. 2020 Disordered Elastic Metasurfaces Phys. Rev. Appl. 13014054

[9] Melde K., Mark A. G., Qiu T. and Fischer P. 2016 Holograms for acoustics Nature 537518 [10]Zhang J., Yang Y., Zhu B., Li X., Jin J., Chen Z., Chen Y. and Zhou Q. 2018 Multifocal point beam forming by a single ultrasonic transducer with 3D printed holograms Appl. Phys. Lett. 113 243502

[11]Tian Y., Wei Q., Cheng Y. and Liu X. 2017 Acoustic holography based on composite metasurface with decoupled modulation of phase and amplitude Appl. Phys. Lett. 110191901

[12]Zhu Y., Hu J., Fan X., Yang J., Liang B., Zhu X. and Cheng J. 2018 Fine manipulation of sound via lossy metamaterials with independent and arbitrary reflection amplitude and phase Nat. Commun. 91632

[13]Brown M. D. 2019 Phase and amplitude modulation with acoustic holograms Appl. Phys. Lett. 115053701

[14]Zhu Y. and Assouar B. 2019 Systematic design of multiplexed-acoustic-metasurface hologram with simultaneous amplitude and phase modulations Phys. Rev. Mater. 3045201

[15]Zuo S., Tian Y., Cheng Y., Deng M., Hu N. and Liu X. 2019 Asymmetric coding metasurfaces for the controllable projection of acoustic images Phys. Rev. Mater. 3065204 [16] Ghaffarivardavagh R., Nikolajczyk J., Glynn Holt R., Anderson S. and Zhang X. 2018 Horn-like space-coiling metamaterials toward simultaneous phase and amplitude modulation Nat. Commun. 9 1349 
[17]Zuo S.-Y., Tian Y., Wei Q., Cheng Y. and Liu X.-J. 2018 Acoustic analog computing based on a reflective metasurface with decoupled modulation of phase and amplitude J. Appl. Phys. 123091704 [18]Zhu X. F. and Lau S. K. 2019 Reflected wave manipulation via acoustic metamaterials with decoupled amplitude and phase Appl. Phys. A 125392

[19]Cheng B., Hou H. and Gao N. 2018 An acoustic metasurface with simultaneous phase modulation and energy attenuation Mod. Phys. Lett. B 321850276

[20]Fink M., Cassereau D., Derode A., Prada C., Roux P., Tanter M., Thomas J.-L. and Wu F. 2000 Time-reversed acoustics Rep. Prog. Phys. 631933

[21]Ma G., Fan X., Sheng P. and Fink M. 2018 Shaping reverberating sound fields with an actively tunable metasurface Proc. Natl. Acad. Sci. USA 1156638

[22]Zhao S. D., Chen A. L., Wang Y. S. and Zhang C. 2018 Continuously Tunable Acoustic Metasurface for Transmitted Wavefront Modulation Phys. Rev. Appl. 10054066

[23]Fan S. W., Zhao S. D., Chen A. L., Wang Y. F., Assouar B. and Wang Y. S. 2019 Tunable Broadband Reflective Acoustic Metasurface Phys. Rev. Appl. 11044038

[24]Chen A. L., Tang Q. Y., Wang H. Y., Zhao S. D. and Wang Y. S. 2020 Multifunction Switching by A Flat Structurally Tunable Acoustic Metasurface for Transmitted Waves Sci. China: Phys., Mech. Astron. 63244611

[25]Fan S. W., Wang Y. F., Cao L., Zhu Y., Chen A. L., Vincent B., Assouar B. and Wang Y. S. 2020 Acoustic vortices with high-order orbital angular momentum by a continuously tunable metasurface Appl. Phys. Lett. 116163504

[26]Yuan S. M., Chen A. L. and Wang Y. S. 2020 Switchable multifunctional fish-bone elastic metasurface for transmitted plate wave modulation J. Sound Vib. 470115168

[27]Fan S. W., Zhao S. D., Cao L., Zhu Y., Chen A. L., Wang Y. F., Donda K., Wang Y. S. and Assouar B. 2020 Reconfigurable curved metasurface for acoustic cloaking and illusion Phys. Rev. B 101024104

[28]Li X. S., Wang Y. F., Chen A. L. and Wang Y. S. 2019 Modulation of out-of-plane reflected waves by using acoustic metasurfaces with tapered corrugated holes Sci. Rep. 915856

[29]Xia J. P., Zhang X. T., Sun H. X., Yuan S. Q., Qian J. and Ge Y. 2018 Broadband Tunable Acoustic Asymmetric Focusing Lens from Dual-Layer Metasurfaces Phys. Rev. Appl. 10014016 [30]Zhu Y., Fei F., Fan S. W., Cao L., Donda K. and Assouar B. 2019 Reconfigurable Origami-Inspired Metamaterials for Controllable Sound Manipulation Phys. Rev. Appl. 12034029 [31]Tian Z., Shen C., Li J., Reit E., Gu Y., Fu H., Cummer S. A. and Huang T. J. 2019 Programmable Acoustic Metasurfaces Adv. Funct. Mater. 291808489

[32]Li X. S., Wang Y. F., Chen A. L. and Wang Y. S. 2020 An arbitrarily curved acoustic metasurface for three-dimensional reflected wave-front modulation J. Phys. D: Appl. Phys. 53195301

[33]Zhou H.-T., Fan S.-W., Li X.-S., Fu W.-X., Wang Y.-F. and Wang Y.-S. 2020 Tunable arc-shaped 
acoustic metasurface carpet cloak Smart Mater. Struct. 29065016

[34]Kinsler L. E., Frey A. R., Coppens A. B. and Sanders J. V. 2000 Fundamentals of Acoustics (John Wiley \& Sons, New York)

[35]Jiang X., Liang B., Li R., Zou X., Yin L. and Cheng J. C. 2014 Ultra-broadband absorption by acoustic metamaterials Appl. Phys. Lett. 105243505

[36]Zhang C. and Hu X. 2016 Three-Dimensional Single-Port Labyrinthine Acoustic Metamaterial: Perfect Absorption with Large Bandwidth and Tunability Phys. Rev. Appl. 6064025

[37] Yang M., Chen S., Fu C. and Sheng P. 2017 Optimal sound-absorbing structures Mater. Horiz. 4 673

[38]Zhu Y., Donda K., Fan S., Cao L. and Assouar B. 2019 Broadband ultra-thin acoustic metasurface absorber with coiled structure Appl. Phys. Express 12114002

[39]Donda K., Zhu Y., Fan S.-W., Cao L., Li Y. and Assouar B. 2019 Extreme low-frequency ultrathin acoustic absorbing metasurface Appl. Phys. Lett. 115173506

[40]Li P., Du Q., Liu M. and Peng P. 2019 Space-coiling acoustic metasurface with independent modulations of phase and amplitude https://arxiv.org/abs/1901.02726

[41] Gao N. and Zhang Y. 2018 A low frequency underwater metastructure composed by helix metal and viscoelastic damping rubber J. Vib. Control 25538

[42]Huang S., Zhou Z., Li D., Liu T., Wang X., Zhu J. and Li Y. 2020 Compact broadband acoustic sink with coherently coupled weak resonances Sci. Bull. 65373

[43]Cao L., Yang Z., Xu Y. and Assouar B. 2018 Deflecting flexural wave with high transmission by using pillared elastic metasurface Smart Mater. Struct. 27075051

[44]Diaz-Rubio A., Asadchy V. S., Elsakka A. and Tretyakov S. A. 2017 From the generalized reflection law to the realization of perfect anomalous reflectors Sci. Adv. 3 e1602714

[45]Díaz-Rubio A. and Tretyakov S. A. 2017 Acoustic metasurfaces for scattering-free anomalous reflection and refraction Phys. Rev. B 96125409

[46]Li Y., Shen C., Xie Y., Li J., Wang W., Cummer S. A. and Jing Y. 2017 Tunable Asymmetric Transmission via Lossy Acoustic Metasurfaces Phys. Rev. Lett. 119035501

[47]Wang X., Fang X., Mao D., Jing Y. and Li Y. 2019 Extremely Asymmetrical Acoustic Metasurface Mirror at the Exceptional Point Phys. Rev. Lett. 123214302

[48]Yoo J. C. and Han T. H. 2009 Fast Normalized Cross-Correlation Circuits Syst. Signal. Process. 28819

[49]Zhu Y., Fan X., Liang B., Cheng J. and Jing Y. 2017 Ultrathin Acoustic Metasurface-Based Schroeder Diffuser Phys. Rev. X 7021034 